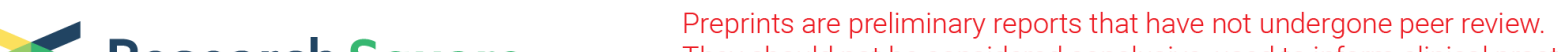 Research Square
or referenced by the media as validated information.
}

\section{Therapeutic Effects and Repair Mechanism of HGF Gene Transfected Mesenchymal Stem Cells on Injured Endometrium via Activating Phosphorylated c-Met}

\section{Xuan Xu}

First Affiliated Hospital of Anhui Medical University https://orcid.org/0000-0003-4166-3399

\section{Jianye Wang}

First Affiliated Hospital of Anhui Medical University

\section{Liu Dong}

First Affiliated Hospital of Anhui Medical University

\section{Qiong Xing}

First Affiliated Hospital of Anhui Medical University

\section{Ying Wang}

First Affiliated Hospital of Anhui Medical University

\section{Ying V. Zhang}

Nuwacell Biotechnologies Co.,Ltd

\section{Yunxia Cao}

First Affiliated Hospital of Anhui Medical University

\section{Ping Zhou}

First Affiliated Hospital of Anhui Medical University

\section{Zhaolian Wei ( $\nabla$ weizhaolian_1@126.com )}

First Affiliated Hospital of Anhui Medical University

\section{Research}

Keywords: Mesenchymal stem cells, Hepatocyte growth factor, Endometrium, Mesenchymal to epithelial transition, HGF/c-Met signaling pathway, Intrauterine adhesion

Posted Date: May 5th, 2021

DOl: https://doi.org/10.21203/rs.3.rs-466396/v1

License: (c) (i) This work is licensed under a Creative Commons Attribution 4.0 International License. Read Full License 


\section{Abstract}

\section{Background}

There are many studies on the advantages of mesenchymal stem cells (MSCs) that could secret various paracrine factors in repairing endometrial injury. It is necessary to improve the stability and effectiveness of MSCs. Hepatocyte growth factor (HGF), as one of the cytokines secreted by MSCs, plays a significant role in vascular repair and mesenchymal to epithelial transformation (MET). It can be deduced that HGF is closely related to the repair process of endometrium.Therefore, we aim to investigate the effect and mechanism of MSCs from umbilical cord transfected with HGF gene in the damaged mouse endometrium.

Methods

HGF gene transfected MSCs were prepared by electroporation. After determining the cell characteristics and cell activity of HGF gene transfected MSCs, the ability of HGF gene transfected MSCs to express HGF was detected by enzyme-linked immunosorbent assay. Totally, 60 female mice were randomly divided into Control group, Saline group, MSCs group, and HGF gene-transfected MSCs (MSCs ${ }^{\text {hg }}$ ) group. Each group of mice received treatment after injury. HE staining were used to evaluate the changes in the thickness of endometrial epithelium and the number of endometrial glands. Immunofluorescence was used to evaluate the molecular repair effect. Real time fluorescent quantitative polymerase chain reaction was used to compare the expression of angiogenesis related factors. Western blot was used to detect the activation of HGF/c-Met and AKT signaling pathways.

Results

HGF gene transfected MSCs retained the characteristics of original MSCs, and the concentration of HGF secreted by MSCs transfected with HGF gene was higher than that of normal MSCs. Compared with normal MSCs, HGF gene transfected MSCs have a more effect in promoting the repair of damaged endometrial epithelium, mainly in significantly increasing the thickness of damaged endometrial epithelium, increasing the number of glands and proliferating cells $(p<0.01)$. Meanwhile, HGF gene transfected MSCs can improve the expression level of endometrial vascular growth related factors and promote the MET process $(p<0.01)$. At the same time, Western blotting confirmed that these repair effects were related to HGF activation of its receptor c-Met and downstream AKT signaling pathway.

\section{Conclusions}

Compared with normal MSCs, HGF gene transfected MSCs have a more significant effect in repairing the damaged endometrial epithelium. This effect is achieved by activating the receptor c-Met of HGF and downstream AKT pathway.

\section{Background}


The healthy endometrium is one of the essential factors in pregnancy, otherwise severe damage of endometrial basement layer in women of childbearing age can cause intrauterine adhesions (IUA) or endometrial thinning, leading to endometrial fibrosis, amenorrhea, infertility or abortion [1-3]. Several therapeutic methods have been tried to improve the regeneration of the endometrium. However, the complex nature of the uterine environment and function limits the possibilities of effective treatment. Therefore, it is an urgent gynecological need to explore novel methods to repair endometrial damage, improve its microenvironment and tolerance status, and enhance the pregnancy rate [4-6].

Previous studies have demonstrated that the administration of mesenchymal stem cells(MSCs)can promote the endometrial regeneration in morphology and function, and the mechanism of endosmetrial regeneration were explored. Accumulated evidences shows that the functional benefit of transplanted MSCs are probably due to the paracrine effect of cytokines produced by transplanted MSCs [7-9]. To maintain the paracrine effect of MSCs, researchers have combined materials or pretreated MSCs with chemical drugs to promote endometrial regeneration after damage [10-12]. However, it may be a more feasible way to enhance the expression of reparative genes in transplanted MSCs to synergistically restore endometrial function after injury.

Hepatocyte growth factor (HGF) is a reparative gene among the numerous cytokines secreted by MSCs $[13,14]$. HGF has a trophic effect on many types of cells, such as epithelial, endothelial and stromal cells, by promoting mitotic activity, growth and migration and preventing apoptosis [15]. It had been explored the combination of cell and gene therapy strategies using MSCs genetically modified to overexpress HGF as a single treatment in some disease model, such as myocardial infarction, hepatocirrhosis, healing of burn wounds, and proved that they have achieved better therapeutic effect then normal MSCs [16-18]. MSCs transfected with HGF can play a better effect in these diseases is precisely because of the function of HGF, which plays an important role in vascular repair and mesenchymal epithelial transformation (MET) process. Similarly, the key mechanisms of endometrial repair are the angiogenesis and MET process. Therefore, MSCs transfected with HGF can enhance the effect of endometrial repair theoretically. In the current study, we transfected MSCs with HGF genes to investigate the beneficial effects in injured endometrial mouse model, and explore the mechanism of its reconstruction and regeneration.

\section{Materials And Methods}

\section{Preparation of HGF gene transfected MSCs}

Human umbilical cords were obtained from full-term cesarean section surgery in The First Affiliated Hospital of Anhui Medical University (Hefei, China). The puerpera had been consented to donate and had signed the informed consent. The MSCs were obtained by the way of umbilical cord tissue climbing and digestive cells adhering to the wall. Briefly, under sterile conditions, umbilical cord tissue was cut along the longitudinal axis, removed the umbilical arteries and vein, peeled the surface membrane gently peeled, and finally rinsed in saline. Cultured the tissue with expansion medium consisting of Dulbecc's modified Eagle's medium (DMEM)/F-12 supplemented with 10\% (v/v) fetal bovine serum (FBS, Gibco,USA). About 
7-10 days later, when the cells cover the culture dish, gently pat the dish to separate the tissue and remove the tissue; the single cell continues to culture and subculture.

MSCs at passage 3 were transfected with the minicircles of HGF (mcHGF) vectors using an electroporation system (Neon Transfection System) according to the manufacturer's instructions. The mcHGF were produced as described by Kay et al (20). MSCs $\left(1.0 \times 10^{6} \mathrm{cells}\right)$ were transfected with mcHGF to generate HGF-expressing cells. Expression of mcHGF in the cells was assessed by fluorescence microscopy at $12 \mathrm{~h}$ post-transfection. The pulse voltage and width conditions were based on the information provided by the Neon Transfection System regarding cell viability and transfection efficacy.

\section{Flow Cytometry (FCM)}

Flow cytometry (FCM) was used to detect whether MSCs retained the characteristics of MSCs after HGF gene transfection. The typical positive markers of MSCs were detected by a flow cytometer (BD FACS Calibur, USA), the specific antibodies for CD73 (1: 1000, Abcam, UK), CD90 (1: 1000, Abcam, UK), and CD105 (1: 1000, Abcam, UK) conjugated with FITC and APC, respectively.

\section{Enzyme-linked immunosorbent assay (ELISA)}

To analyse the amount of HGF expressed by transfected cells, the culture media of MSCs transfected with the indicated minicircle vectors was analysed by an enzyme-linked immunosorbent assay (ELISA) at $24 \mathrm{~h}, 48 \mathrm{~h}, 72 \mathrm{~h}$ and $96 \mathrm{~h}$ post-transfection. The levels of HGF were quantified using a human HGFR Platinum ELISA (Affymetrix, San Diego, CA, USA), according to the manufacturer's instructions. After applying stop solution, absorbance was measured at $450 \mathrm{~nm}$.

\section{Establishment of the mouse endometrial injury model}

Eight-week-old female C57BL/6 mouse(SPF level, Wei Tong Li Hua)were used in the study. They were housed five to six mouse per cage at room temperature,with humidity $40-60 \%$ light/dark cycle $12 \mathrm{~h} / 12 \mathrm{~h}$, and free access to food and water. The mice were anesthetized and the uterine horns were exteriorized through a midline incision under sterile surgical conditions, then injected about 50ul 95\% ethanol until filling one side of the uterine horns, rinsed with saline three time nextly, repositioned the uterus gently then sutured the wound and waited for anesthesia to wake up. Ethanol-treated experimental and saline-treated control mouse were examined after 5 days to validate the establishment of the endometrial injury model.

\section{Groups and treatment}

For the in-vivo experiment, 8-week-old female mice were randomly divided into four groups. Each group consisted of 15 mice, a total of 60 mice with synchronous estrous cycle. The groups are as follows: Control group (did not undergo any treatment), Saline group (saline injected post-injury group), MSCs group (MSC injected post-injury group), and HGF gene transfected MSCs (MSCs ${ }^{\text {hg }}$ ) group (HGF gene transfected MSCs injected post-injury group). Cells $\left(2 \times 10^{5}\right)$ were injected in a total volume of $200 \mathrm{ul}$, and were injected into the tail vein on the fifth day after injury of mouse endometrium. Consider the half-life of living cells in vivo, we select to execute the mouse one week after cell transplantation and take out the 
uterine tissue. Additionally, we confirmed that all experiments and methods were performed in accordance with the relevant ethical guidelines and regulations, and they were approved by the Experimental Center of the First Affiliated Hospital of Anhui Medical University.

\section{Hematoxylin and eosin (H\&E) staining}

One estrous period after treatment, the "Y" uterus was completely removed from the abdominal cavity. The tissues were paraffin embedded, sectioned (4um) and stained with hematoxylin and eosin (H\&E). The morphological changes of mouse uterus were observed by inverted phase contrast microscope. The thickness of endometrial epithelium and the number of glands were recorded. Select 10 fields in each image to count.

\section{Immunofluorescence analysis}

Sections of mouse uterus ( $\mathrm{n}=5$ per time point) were fixed in pre-cooled acetone and incubated with $0.3 \%$ hydrogen peroxide prepared in $10 \%$ methanol for 15 min. After washing with PBS, the sections were blocked using an Avidin/Biotin Blocking Kit (Vector Laboratories, CA, USA). After incubation, blocking was performed following the manufacturer's instructions of Mouse on Mouse Fluorescein Kit (Vector Laboratories). Sections were incubated with a mouse anti-h-Nuclei monoclonal antibody (Cell Signaling, USA) overnight. The next day, sections were incubated with rabbit anti-mouse IgG for $1 \mathrm{~h}$. After DAPI staining, sections were observed with a confocal microscope (Carl Zeiss LSM700, Prenzlauer, Berlin, Germany).

\section{Quantitative reverse-transcription polymerase chain reaction (qRT-PCR)}

Total RNA from rat uteri was extracted using Trizol (Invitrogen, Carlsbad, CA, USA) according to the manufacturer's protocols; $1 \mu \mathrm{g}$ of total RNA was subjected to reverse transcription of mRNA using oligo dT as a primer and a reverse transcription kit (Transgen Biotech, Beijing, China) to generate total cDNA. The quantitative PCR was then carried using primers shown in Table 1 and faststart universal SYBR Green master (Toyobo, Osaka, Japan) and 7500 real-time PCR system (Applied Biosystems company, foster, California, USA) were used for quantitative PCR. $\beta$-actin is used for standardization. The experiment was repeated at least three times. 
Table 1

Primer sequences

\begin{tabular}{|lll|}
\hline Gene name & Primer sequence \\
\hline VEGF & Forward 5'-3' & $5^{\prime}$ - TAGAGTACATCTTCAAGCCGTC - 3' \\
& reverse 5'-3' & $5^{\prime}$ - CTTTCTTTGGTCTGCATTCACA-3' \\
\hline Forward 5'-3' & $5^{\prime}$ - TATGCTGTTTGAACTTATGCGC - 3' \\
\hline reverse 5'-3' & $5^{\prime}$ - GTTCTCCTCGCTGTAGTAGAAG - 3' \\
& Forward 5'-3' & $5^{\prime}$ - GTACGCCAACACAGTGCTGTC-3' \\
\hline & reverse 5'-3' & $5^{\prime}$ - GCTCAGGAGGAGCAATGATCTTG - 3' \\
\hline
\end{tabular}

\section{Western blotting (WB)}

Frozen uterus samples were homogenised with tissue extraction buffer inhibitors (Beyotime, China), and the resulting lysates were centrifuged at $12,000 \mathrm{~g}$ at $4^{\circ} \mathrm{C}$ for $10 \mathrm{~min}$. The supernatants were collected, and the protein concentration was measured. The supernatants were denatured in sample buffer and heated in boiling water for $5 \mathrm{~min}$. Proteinswere separated by $10 \%$ SDS-PAGE and transferred electrophoretically from the gels to polyvinylidene difluoride (PVDF) transfer membranes. The membranes were incubated for $2 \mathrm{~h}$ in a blocking solution containing 5\% skim milk and 0.05\% Tween-20 in PBS (PBS-Tween). The membranes were washed briefly in PBS-Tween and incubated with the c-Met (1:1000, Cell Signaling, USA), p-c-Met (1:1000, Cell Signaling, USA), AKT (1:1000, Cell Signaling, USA), and p-AKT (1:1000, Cell Signaling, USA) antibody at $4{ }^{\circ} \mathrm{C}$ overnight. The membranes were next washed 3 times in PBS-Tween by using a rotary shaker. The washed membranes were incubated with horseradish peroxidase- (HRP-) conjugated anti-rabbit for $1 \mathrm{~h}$. The membranes were washed again and processed with an ECL detection kit (Biosharp, USA) according to the manufacturer's direction to visualize the proteins recognized by the antibodies.

\section{Statistical analysis}

SPSS software was used for statistical analysis. All data are expressed as standard deviation of at least three independent operations. The quantitative data are expressed as the mean standard deviation of at least three independent experiments. Statistically significant is $P<0.05$. The asterisk $(* \star)$ indicates $P<$ 0.01 .

\section{Results}

\section{Characteristics of HGF gene-transfected MSCs}

To characterize the HGF gene transfected MSCs, we detected the morphology and cell proliferation of MSCs and HGF gene transfected MSCs. Figure 1A shows the cell morphology of MSCs and HGF gene transfected MSCs, They have the same shape of spindle cells. Under the same culture conditions, MSCs 
and HGF gene transfected MSCs were almost the same cell proliferation (Fig. 1B). To verify the characteristics of mesenchymal stem cells, we checked the positive markers, including CD73, CD90 and CD105, were both highly expressed in MSCs and HGF gene transfected MSCs (Fig. 1C). To assess the effect of HGF gene transfection, the concentration of HGF gene were detected. The concentration of HGF in the cell suspension of HGF gene transfected MSCs was higher than that of the non-transfected MSCs at 0, 1, 2, 3 and 4 day after transfection (Fig. 1D). Compared with the non-transfected MSCs, the HGF gene transfected MSCs progressively increased HGF level at 1,2 and 3 day. Non-transfected MSCs gradually increased but still remained totally lower than 4 day. These results indicated that HGF gene transfected MSCs retain the characteristics of original MSCs, and significantly increased the expression of HGF gene in transfected MSCs.

\section{HGF gene transfected MSCs recover the morphology of injured uterus}

In order to verify the effect of HGF gene transfected MSCs, we established the mouse model of endometrial injury, and observed the morphological characteristics of mouse uterus after HGF gene transfected MSCs therapy. The results show that HGF gene transfected MSCs could improve the appearance and morphological characteristics of the uterus on the damaged side, and the appearance of the uterus is close to the normal side (Fig. 2A). H \& E staining showed that the endometrial structure of the HGF gene transfected MSCs group was relatively complete, the epithelial cells were closely arranged, and the glands were clearly visible. In the Saline group, the endometrium of the injured side was poor, the thickness of endometrium was obviously thinner, and the epithelium was discontinuous and incomplete (Fig. 2B). The thickness of endometrial epithelium and the number of endometrial glands in each group were counted. HGF gene transfected MSCs treatment group was significantly higher than those of the Saline group and MSCs group. Morphologically, HGF gene transfected MSCs is more effective than normal MSCs. (Fig. 2C, D).

\section{HGF gene transfected MSCs improve proliferation and decrease apoptosis of endometrial cells}

In order to verify the repair ability of HGF, we detected the proliferation and apoptosis of epithelial cells. It can be seen that HGF gene transfected MSCs can improve the proliferation ability of damaged endometrial epithelial cells (Fig. 3A). Through the statistical analysis of Ki-67 fluorescence intensity, a relatively quantitative comparison can reflect the therapeutic effect of HGF gene transfected MSCs, who was significantly higher than those of the Saline group and MSCs group (Fig. 3B). Besides, HGF gene transfected MSCs can reduce the apoptosis of endometrial cells (Fig. 3C). Through the statistical analysis of TUNEL fluorescence intensity, the therapeutic effect of HGF gene transfected MSCs can be quantitatively reflected. It is significant that the apoptotic cells in HGF gene transfected MSCs group were less than Saline group and MSCs group (Fig. 3D). 


\section{HGF gene transfected MSCs improves the angiogenesis of damaged endometrium}

In order to verify the repair effect of HGF on the injured endometrial vessels, we detected the expression levels of two kinds of angiogenesis related mRNA: vascular endothelial growth factor (VEGF) and insulinlike growth factor 1 (IGF-1). It can be found that HGF gene-transfected MSCs can improve the angiogenesis of endometrial epithelial cells. The mRNA expression of VEGF and IGF-1 in uterus of each group was counted (Fig. 4A, B). It can be confirmed that HGF gene transfected MSCs have better vascular repair ability than normal MSCs.

\section{HGF gene transfected MSCs promote the MET process in damaged endometrium}

To observe the effect of HGF gene transfected MSCs on mesenchyma-lepithelial transition process in damaged endometrium, we used immunofluorescence to label epithelial marker E-cadherin and mesenchymal marker Vimentin respectively (Fig. 5A,B). By observing the expression of E-cadherin and Vimentin in epithelial and stromal regions, we quantitatively analyzed their fluorescence intensity, and counted the ratio of E-cadherin and Vimentin intensity in each treatment group (Fig. 5C). The results showed that the process of MET in HGF gene transfected MSCs group was more active and the degree of epithelial transformation was higher. These results show that HGF plays an indispensable role in MET process.

HGF gene transfected MSCs plays a role by activating phosphorylated c-Met and downstream phosphorylated AKT pathway

Finally, we explore how HGF can play its role. The first step is to verify the HGF receptor c-Met, detect the expression level of c-Met total protein and its phosphorylation(p-c-Met)by Western blot, and then check the expression level of AKT and AKT phosphorylation $\square$ p-AKTDin its downstream pathway (Fig. 6A,B) The results showed that the expression of p-c-Met in HGF gene transfected MSCs group was the highest in acute inflammation stage after injury, and the expression of $\mathrm{p}-\mathrm{AKT}$ in its downstream pathway was also the highest.

\section{Discussion}

In this study, we found that HGF gene transfected MSCs had better therapeutic effect compared with normal MSCs in mice model of endometrial damage. Increasing evidence suggests that the therapeutic effects of MSCs mainly depend on their capacity to secrete a variety of growth factors and cytokines [19], including HGF. However, exogenous HGF have a temporary effect on the tissue regeneration, due to its short half-life in the blood circulation[20]. In contrast, MSCs can persistently release HGF, which ensures a continuous expression of HGF in the organisms and overcomes the disadvantage of only injecting exogenous HGF. In that regard, genetic engineering of MSCs transfected with HGF gene can improve the 
intrinsic secretion of HGF, thus improving the endometrial repair potential of these cells in a context of gene therapy.

It is particularly important to generate the ideal HGF gene transfected MSCs. Even though some viral vectors were confirmed to be safe for clinical use, an improved method that can entirely avoid the integration of viral vectors is required. In this study, we generated HGF-secreting MSCs using minicircle vectors containing only the transgene expression cassette [21-23]. Minicircles are small circular plasmids that carry transgenes. The absence of the bacterial backbone and the non-integrating transfection system make minicircle vectors become an attractive delivery tool in vivo for future clinical applications. Genetically engineering HGF into MSCs by minicircles to treat injured endometrium would be optimal from both pharmacological and practical perspectives [24, 25]. After transfected with HGF gene, we detected characteristics and secretion ability of cells to ensure its safety and effectiveness. By analyzing the cell activity of MSCs after HGF gene transfection and the characteristic expression of mesenchymal stem cell markers (CD73, CD90 and CD105), it was confirmed that HGF gene transfection would not change the inherent properties of MSC. At the same time, the ability of MSCs to secrete HGF before and after transfection was further tested. It was found that the ability of MSC to secrete HGF after transfection was significantly improved. In line with our expectations, HGF gene transfected MSCs can be used for transplantation therapy. In order to verify the therapeutic effect of MSCs transfected with HGF gene on damaged endometrium, we compared the treatment results of ordinary MSCs and HGF gene transfected MSCs in mouse model through biochemical, histological and molecular biological methods. The results show that HGF gene transfected MSCs has a positive recovery effect on endometrial regeneration, which is more significant than ordinary MSCs.

Recent studies have shown that the endometrial tissue of patients with uterine trauma is characterized by vascular closure and microvascular damage, angiogenesis will appear in endometrial tissue after treatment, suggesting that angiogenesis of damaged endometrium may affect the regeneration of endometrium [26, 27]. Meanwhile, HGF plays an indispensable role in angiogenesis [28, 29]. In order to explore the mechanism of HGF gene transfected MSCs in the treatment of endometrial injury, we verified the ability of vascular regeneration after transplanted with normal MSCs and HGF gene transfected MSCs. In our experiment, the expression of VEGF and IGF-1 in the endometrium of mice treated with MSCs transfected with HGF gene was higher than that of mice treated with normal MSCs. This further suggests that HGF gene transfected MSCs can play a role in angiogenesis by increasing the amount of angiogenesis related factors.

Another role of HGF plays is about mesenchymal to epithelial transition (MET) process. First and foremost, it absolutely believed that HGF and its receptor c-Met related to the transformation of the epithelium and mesenchyme, most of these reports are about tumors or endometriosis [30-33]. The endometrium is the dynamic inner layer of the uterus, from a functional point of view, the endometrium is composed of functional layer and basement layer, and from the perspective of histology, composed of mesenchymal and epithelial cells that undergo monthly proliferation, differentiation, and shedding throughout the menstrual cycle in childbearing age women. In the functional layer of endometrium, the 
epithelium and mesenchyme are in a dynamic balance process. When the epithelial tissue of endometrium is damaged, the mesenchyme will transform to the epithelium, so as to maintain the integrity of the epithelium on the surface of endometrium [34,35]. Furthermore, MSCs transfected with HGF gene can promote the transformation of mesenchymal to epithelial. The expression of the related antigen, E-cadherin in epithelial cells and $\mathrm{N}$-cadherin in mesenchymal cells can reflect the degree of MET [36]. Therefore, we detected the expression of protein to verify that MSCs transfected with HGF plays a role by binding to its receptor c-Met, activating the phosphorylation of c-Met receptor and activating the downstream AKT pathway. This process plays a leading role in both increasing angiogenesis and MET process [37-39]. However, preliminary detection of these two proteins only confirmed that HGF transfected MSCs plays a role in the repair of endometrium through angiogenesis and mesenchyme to epithelium transformation, and the more exact mechanism is still unclear.

\section{Conclusion}

In summary, the present study shows that cell therapy with HGF gene transfected MSCs significantly promotes endometrial repair in the morphological and molecular aspects, these effects are superior to treatment with normal MSCs. In addition, the enhanced therapeutic effects of HGF gene transfected MSCs were accompanied by increased levels of the proliferative cell, and decreased the apoptosis. Moreover, MSCs transfected with HGF gene can promote angiogenesis and MET process by activating $\mathrm{p}$ c-Met pathway after transplantation. These results suggest that cell therapy with HGF gene transfected MSCs may be a new and effective tool for the treatment of injured endometrium.

\section{Abbreviations}

HGF: Hepatocyte growth factor;

IUA: Intrauterine adhesion;

IGF-1: Insulin-like growth factor 1;

MET: Mesenchymal to epithelial transition;

MSC: Mesenchymal stem cell;

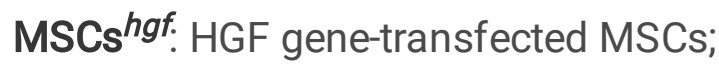

p-c-Met: phosphorylation of c-Met;

p-AKT: phosphorylation of AKT;

VEGF: Vascular endothelial growth factor.

\section{Declarations}




\section{Data Availability}

The data that support the findings of this study are available upon request from the corresponding author. The data are not publicly available due to privacy concerns or ethical reasons.

\section{Acknowledgements}

The authors thank the Core Facility Center for Life Sciences, Anhui Agricultural University for their support.

\section{Funding}

This work was supported by grants from National Natural Science Foundation of China (No. 81901437), Anhui Provincial Natural Science Foundation (No.1908085QH314).

Author information

\section{Affiliations}

Reproductive Medicine Center, Department of Obstetrics and Gynecology, the First Affiliated Hospital of Anhui Medical University, No 218 Jixi Road, Hefei 230022, Anhui, China.

Xuan Xu, Jianye Wang, Liu Dong, Qiong Xing, Ying Wang, Ping Zhou, Yunxia Cao, Zhaolian Wei Anhui Province Key Laboratory of Reproductive Health and Genetics, Anhui Medical University, No 81 Meishan Road, Hefei 230032, Anhui, China.

Xuan Xu, Jianye Wang, Liu Dong, Yunxia Cao, Zhaolian Wei

NHC Key Laboratory of study on abnormal gametes and reproductive tract (Anhui Medical University), No 81 Meishan Road, Hefei 230032, Anhui, China.

Xuan Xu, Jianye Wang, Liu Dong, Yunxia Cao, Zhaolian Wei

Anhui Province Key Laboratory of Reproductive Health and Genetics, No 81 Meishan Road, Hefei 230032, Anhui, China

Qiong Xing, Ying Wang, Ping Zhou, Yunxia Cao, Zhaolian Wei

Nuwacell Biotechnologies Co., Ltd, No 2800 Chuangxin Avenue, Hefei 230032, Anhui, China

Ying V. Zhang

\section{Authors' Contributions}

X.X. and J.W. contributed equally to this work. Z.W. and Y.Z. designed and supervised the study. X.X., J.W. and L.D. collected materials, performed the experiments, analyzed the data, and wrote the manuscript. All 
authors wrote parts of the manuscript, read and approved the final manuscript.

\section{Corresponding authors}

Correspondence to Zhaolian Wei or Ying V. Zhang.

\section{Availability of data and materials:}

All data generated or analyzed during this study are included in this published article.

\section{Ethics approval and consent to participate:}

This study was approved by the Hospital Ethics Committee of the First Affiliated Hospital of Anhui Medical University.

\section{Consent for publication:}

Not applicable.

\section{Competing interests:}

The authors declare that they have no competing interests.

\section{References}

1. Deans R, Abbott J. Review of intrauterine adhesions. J Minim Invasive Gynecol. 2010; 17:555-69.

2. Zupi E, Centini G, Lazzeri L. Asherman syndrome: an unsolved clinical definition and management. Fertil Steril. 2015; 104:1380-1.

3. March CM. Management of Asherman's syndrome. Reprod Biomed Online. 2011; 23:63-76.

4. Zhao M, Chang C, Liu Z, Chen LM, Chen Q. Treatment with low-dose aspirin increased the level LIF and integrin beta3 expression in mice during the implantation window. Placenta. 2010; 31:1101-5.

5. Zhang L, Li Y, Guan CY, Tian S, Lv XD, Li JH, et al. Therapeutic effect of human umbilical cord-derived mesenchymal stem cells on injured rat endometrium during its chronic phase. Stem Cell Res Ther. 2018; 9:36.

6. Yang H, Wu S, Feng R, Huang J, Liu L, Liu F, et al. Vitamin C plus hydrogel facilitates bone marrow stromal cell-mediated endometrium regeneration in rats. Stem Cell Res Ther. 2017; 8:267.

7. Jing Z, Qiong Z, Yonggang W, Yanping L. Rat bone marrow mesenchymal stem cells improve regeneration of thin endometrium in rat. Fertil Steril. 2014; 101:587-94.

8. Gan L, Duan H, Xu Q, Tang YQ, Li JJ, Sun FQ, et al. Human amniotic mesenchymal stromal cell transplantation improves endometrial regeneration in rodent models of intrauterine adhesions. Cytotherapy. 2017; 19:603-16. 
9. Zhao G, Cao Y, Zhu X, Tang X, Ding L, Sun H, et al. Transplantation of collagen scaffold with autologous bone marrow mononuclear cells promotes functional endometrium reconstruction via downregulating DeltaNp63 expression in Asherman's syndrome. Sci China Life Sci. 2017; 60:404-16.

10. Li X, Sun H, Lin N, Hou X, Wang J, Zhou B, et al. Regeneration of uterine horns in rats by collagen scaffolds loaded with collagen-binding human basic fibroblast growth factor. Biomaterials. 2011; 32:8172-81.

11. Campo H, Cervello I, Simon C. Bioengineering the Uterus: An Overview of Recent Advances and Future Perspectives in Reproductive Medicine. Ann Biomed Eng. 2017; 45:1710-7.

12. Pourjafar M, Saidijam M, Mansouri K, Ghasemibasir H, Karimi Dermani F, Najafı R. All-trans retinoic acid preconditioning enhances proliferation, angiogenesis and migration of mesenchymal stem cell in vitro and enhances wound repair in vivo. Cell Prolif. 2017; 50.

13. Bell LN, Cai L, Johnstone BH, Traktuev DO, March KL, Considine RV. A central role for hepatocyte growth factor in adipose tissue angiogenesis. Am J Physiol Endocrinol Metab. 2008; 294:E336-44.

14. Inagaki Y, Higashi K, Kushida M, Hong YY, Nakao S, Higashiyama R, et al. Hepatocyte growth factor suppresses profibrogenic signal transduction via nuclear export of Smad3 with galectin-7. Gastroenterology. 2008; 134:1180-90.

15. Ding S, Merkulova-Rainon T, Han ZC, Tobelem G. HGF receptor up-regulation contributes to the angiogenic phenotype of human endothelial cells and promotes angiogenesis in vitro. Blood. 2003; 101:4816-22.

16. Zhang Y, Li R, Rong W, Han M, Cui C, Feng Z, et al. Therapeutic effect of hepatocyte growth factoroverexpressing bone marrow-derived mesenchymal stem cells on CCl4-induced hepatocirrhosis. Cell Death Dis. 2018; 9:1186.

17. Gomez-Mauricio G, Moscoso I, Martin-Cancho MF, Crisostomo V, Prat-Vidal C, Baez-Diaz C, et al. Combined administration of mesenchymal stem cells overexpressing IGF-1 and HGF enhances neovascularization but moderately improves cardiac regeneration in a porcine model. Stem Cell Res Ther. 2016; 7:94.

18. Lai L, Chen J, Wei X, Huang M, Hu X, Yang R, et al. Transplantation of MSCs Overexpressing HGF into a Rat Model of Liver Fibrosis. Mol Imaging Biol. 2016; 18:43-51.

19. Zhao S, Qi W, Zheng J, Tian Y, Qi X, Kong D, et al. Exosomes Derived from Adipose Mesenchymal Stem Cells Restore Functional Endometrium in a Rat Model of Intrauterine Adhesions. Reprod Sci. 2020; 27:1266-75.

20. Sivasami Pulavendran, Rose $\mathbb{C}$, Mandal AB. Hepatocyte growth factor incorporated chitosan nanoparticles augment the differentiation of stem cell into hepatocytes for the recovery of liver cirrhosis in mice. Journal of Nanobiotechnology. 2011; 9.

21. Jones $\mathrm{CH}$, Chen $\mathrm{CK}$, Ravikrishnan A, Rane S, Pfeifer BA. Overcoming nonviral gene delivery barriers: perspective and future. Mol Pharm. 2013; 10:4082-98.

22. Eugene H.Kaji M, Jeffrey M. Leiden M, PhD. Gene and Stem Cell Therapies. JAMA. 2001; 285:545-50. 
23. Kim MD, Kim SS, Cha HY, Jang SH, Chang DY, Kim W, et al. Therapeutic effect of hepatocyte growth factor-secreting mesenchymal stem cells in a rat model of liver fibrosis. J Exp Mol Med. 2014; 46:e110.

24. Park N, Rim YA, Jung H, Kim J, Yi H, Kim Y, et al. Etanercept-Synthesising Mesenchymal Stem Cells Efficiently Ameliorate Collagen-Induced Arthritis. Sci Rep. 2017; 7:39593.

25. Kay MA, He CY, Chen ZY. A robust system for production of minicircle DNA vectors. Nat Biotechnol. 2010; 28:1287-9.

26. Chen $\nabla Y$, Chang $\nabla Y$, Yao S. Role of angiogenesis in endometrial repair of patients with severe intrauterine adhesion. Int J Clin Exp Pathol 2013; 6:1343-50.

27. Demir R, Yaba A, Huppertz B. Vasculogenesis and angiogenesis in the endometrium during menstrual cycle and implantation. Acta Histochemica. 2010; 112:203-14.

28. Agostinis C, Masat E, Bossi F, Ricci G, Menegazzi R, Lombardelli L, et al. Transcriptomics and Immunological Analyses Reveal a Pro-Angiogenic and Anti-Inflammatory Phenotype for Decidual Endothelial Cells. Int J Mol Sci. 2019; 20.

29. Patki S, Patki U, Patil R, Indumathi S, Kaingade P, Bulbule A, et al. Comparison of the levels of the growth factors in umbilical cord serum and human milk and its clinical significance. Cytokine. 2012; 59:305-8.

30. Jiao D, Wang J, Lu W, Tang X, Chen J, Mou H, et al. Curcumin inhibited HGF-induced EMT and angiogenesis through regulating c-Met dependent PI3K/Akt/mTOR signaling pathways in lung cancer. Molecular Therapy - Oncolytics. 2016; 3.

31. Jiao D, Chen J, Li Y, Tang X, Wang J, Xu W, et al. miR-1-3p and miR-206 sensitizes HGF-induced gefitinib-resistant human lung cancer cells through inhibition of c-Met signalling and EMT. Journal of Cellular and Molecular Medicine. 2018; 22:3526-36.

32. Liu X, Shen M, Qi Q, Zhang H, Guo SW. Corroborating evidence for platelet-induced epithelialmesenchymal transition and fibroblast-to-myofibroblast transdifferentiation in the development of adenomyosis. Hum Reprod. 2016; 31:734-49.

33. Khan KN, Kitajima M, Hiraki K, Fujishita A, Nakashima M, Masuzaki H. Involvement of hepatocyte growth factor-induced epithelial-mesenchymal transition in human adenomyosis. Biol Reprod. 2015; $92: 35$.

34. Mihm M, Gangooly S, Muttukrishna S. The normal menstrual cycle in women. Anim Reprod Sci. $2011 ; 124: 229-36$.

35. Owusu-Akyaw A, Krishnamoorthy K, Goldsmith LT, Morelli SS. The role of mesenchymal-epithelial transition in endometrial function. Hum Reprod Update. 2019; 25:114-33.

36. Wong SHM, Fang CM, Chuah LH, Leong CO, Ngai SC. E-cadherin: Its dysregulation in carcinogenesis and clinical implications. Crit Rev Oncol Hematol. 2018; 121:11-22.

37. Hong-Qing Zhuang, Hongxia Zhuang, QifuBo4, YihangGuo, Wang® J, Lu-JunZhao®, et al. Experimental study on the regulation of erlotinib-induced radiosensitization with an anti-c-MET monoclonal antibody. Cancer Cell International 2014; 14. 
38. Zhuang HQ, Bo QF, Yuan ZY, Wang J, Zhao LJ, Wang P. The different radiosensitivity when combining erlotinib with radiation at different administration schedules might be related to activity variations in c-MET-PI3K-AKT signal transduction. Onco Targets Ther. 2013; 6:603-8.

39. Brigitte Boyer, Valle's $\triangle A M$, Edme N. Induction and Regulation of Epithelial-Mesenchymal transitions. Biochemical Pharmacology. 2000; 60:1091-9.

\section{Figures}

A

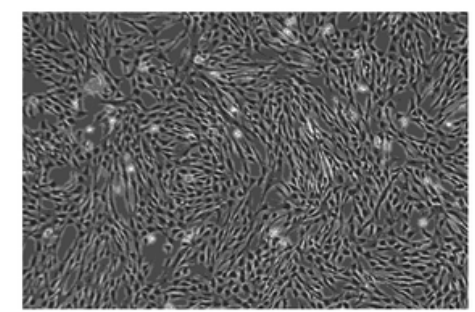

Day3 MSCs

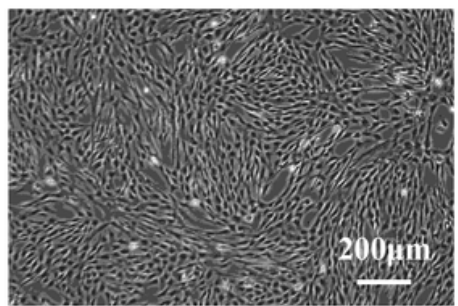

Day3 MSCs ${ }^{\text {her }}$
C

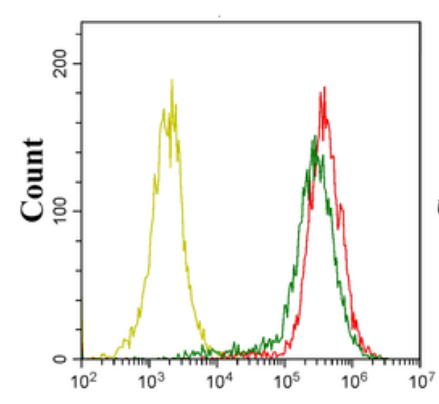

CD90-FITC-A

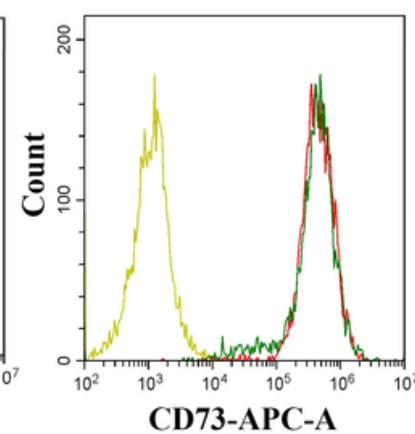

CD73-APC-A

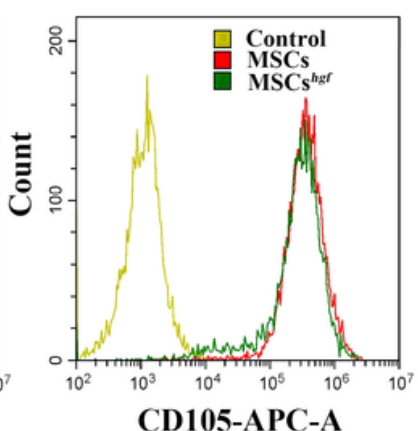

B

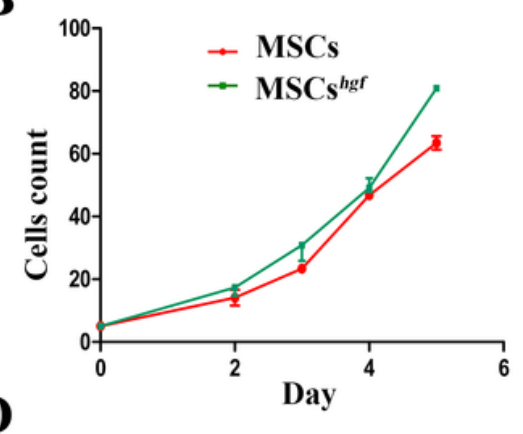

D

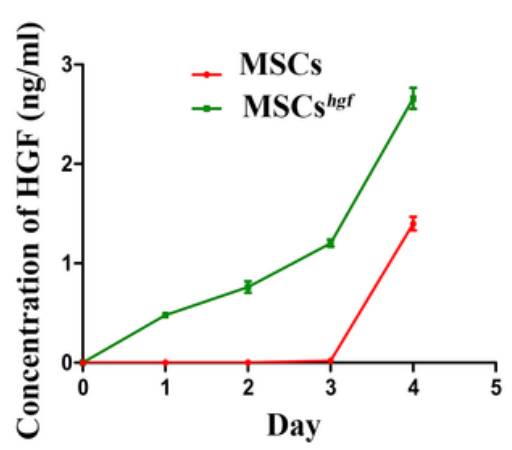

\section{Figure 1}

HGF gene transfected MSCs (MSCshgf in the legend) retain the characteristics of original MSCs, and significantly increased the expression of HGF gene in transfected MSCs. A. The cell morphology of MSCs and HGF gene transfected MSCs. B. Cell proliferation of MSCs and HGF gene-transfected MSCs. C. Positive markers (CD73, CD90 and CD105) on the surface of mesenchymal stem cells were tested in MSCs and HGF gene-transfected MSCs. D. The expression of HGF in MSCs and HGF gene transfected MSCs. 


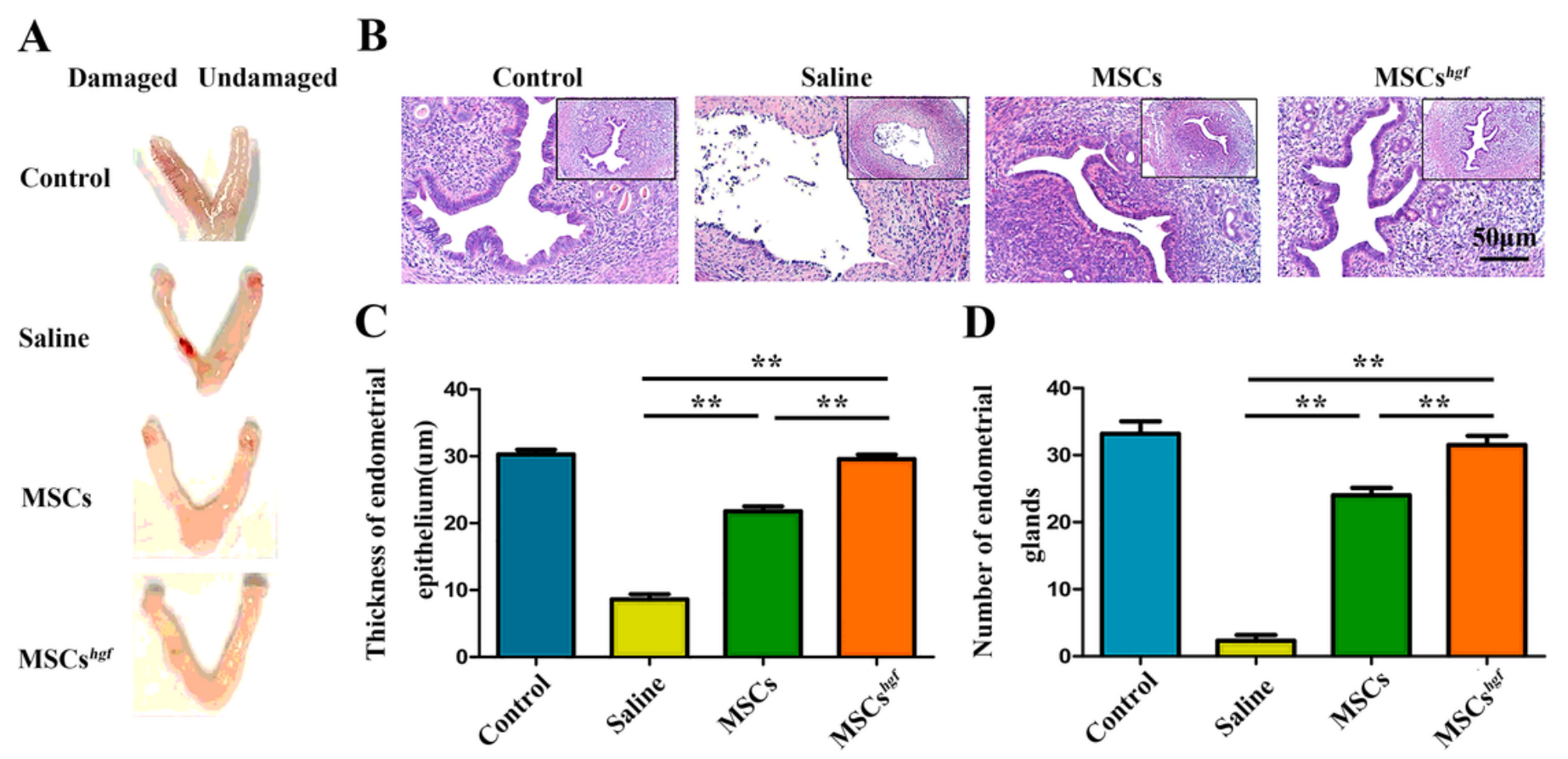

Figure 2

HGF gene transfected MSCs improve the morphological characteristics and changes of uterus. A. The mouse uterus tissue samples were taken out. B. H \& E staining of mouse uterus $(40 \times)$. C. The difference of the thickness of endometrial epithelium in each treatment group. D. The difference of the number of glands in each treatment group. ${ }^{*} \mathrm{p}<0.01$. 
A

MSCs
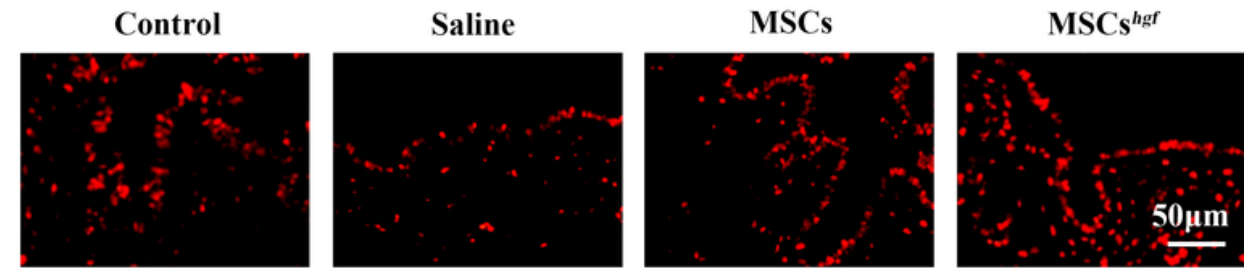

C
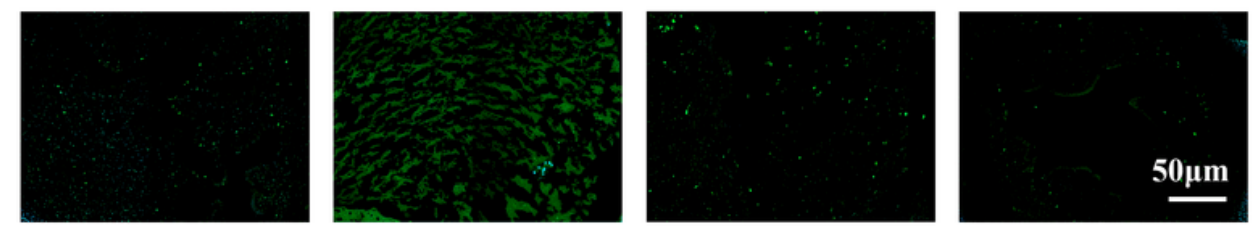

B
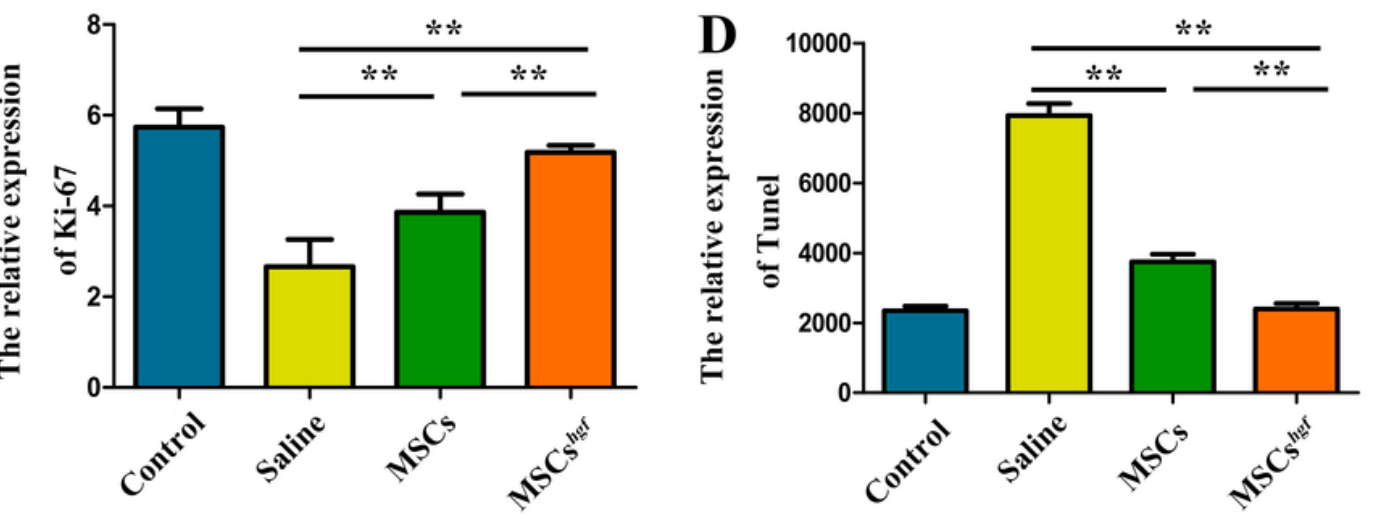

\section{Figure 3}

HGF gene-transfected MSCs increase proliferation and decrease apoptosis of endometrial cells in uterus. A. The expression of $\mathrm{Ki}-67$ immunofluorescence in each treatment group. B. The statistical analysis of Ki67 immunofluorescence intensity in each treatment group. C. The expression of Tunel immunofluorescence in each treatment group. D. The statistical analysis of Tunel immunofluorescence intensity in each treatment group. ${ }^{\star \star} p<0.01$. 


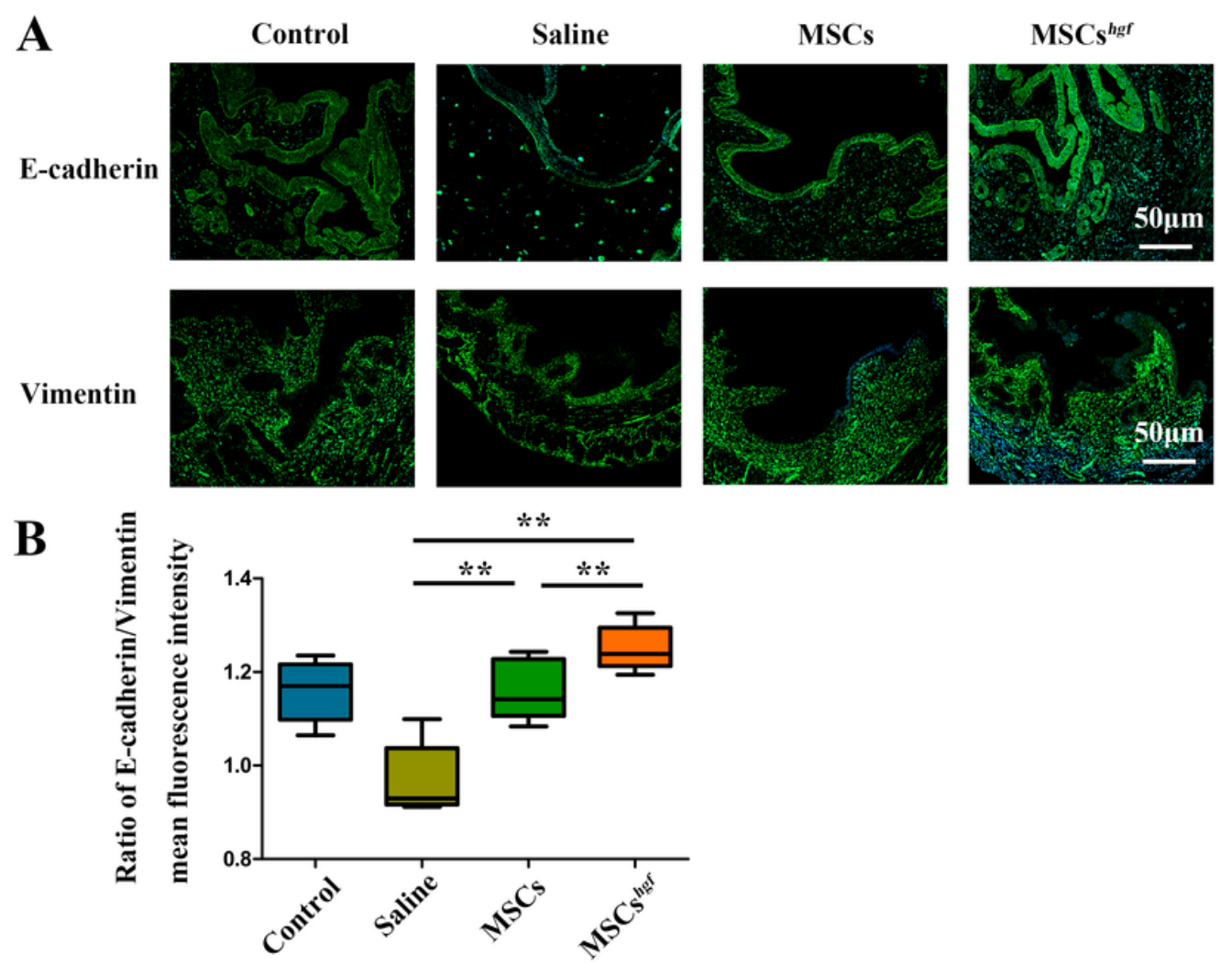

Figure 4

HGF gene-transfected MSCs increase the angiogenesis of damaged endometrium. A. The expression of VEGF mRNA in each treatment group. B. The expression of IGF-1 mRNA in each treatment group. $\star * p<0.01$. 

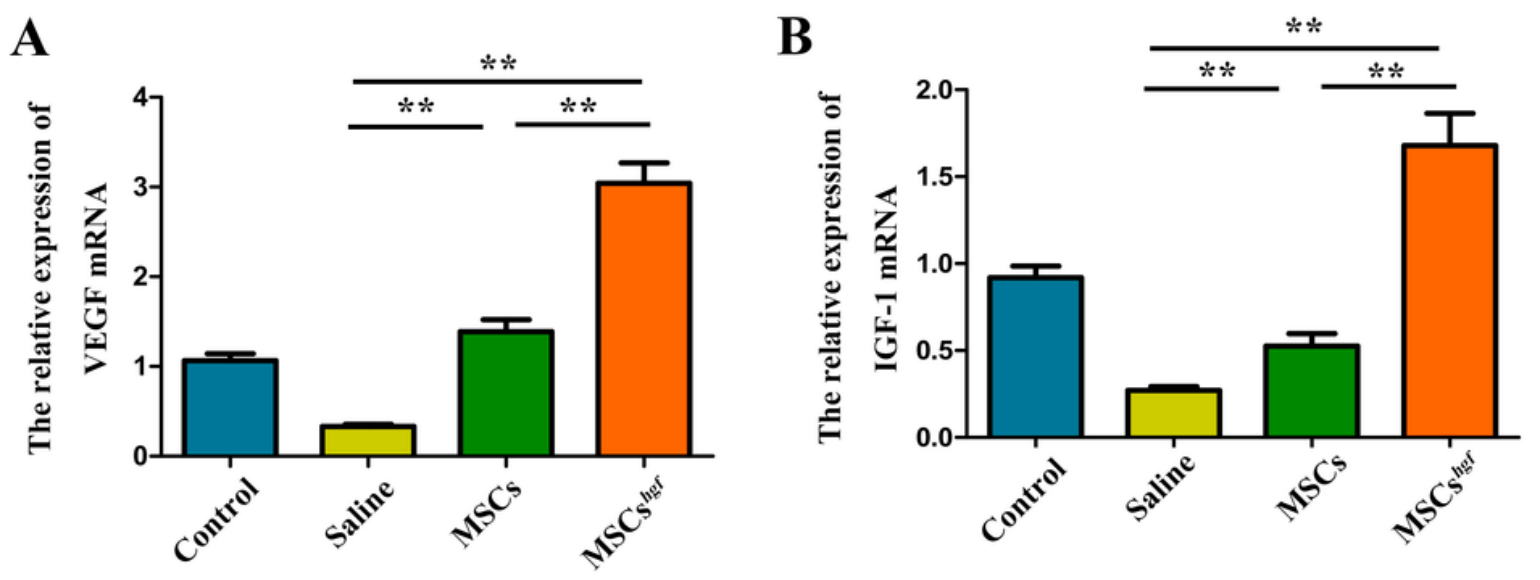

Figure 5

HGF gene-transfected MSCs promote the degree of MET process in damaged endometrium. A. The expression of E-cadherin immunofluorescence in each treatment group. B. The expression of Vimentin immunofluorescence in each treatment group. C. The ratio of E-cadherin and Vimentin intensity in each treatment group. ${ }^{\star \star} p<0.01$. 
A

B

p-c-Met

c-Met

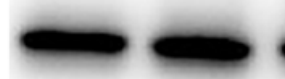

$\beta$-actin

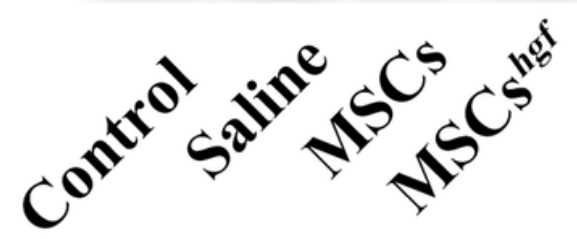

p-AKT

AKT

$\beta$-actin

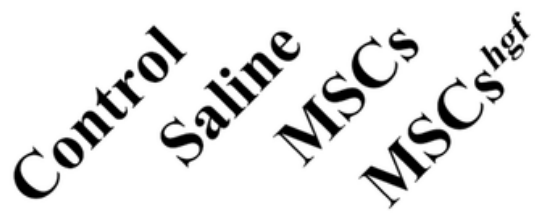

Figure 6

HGF gene-transfected MSCs plays a role by activating phosphorylated c-Met and downstream phosphorylated AKT pathway. A. The expression of p-c-Met in each treatment group. B. The expression of $\mathrm{p}$-AKT in each treatment group. 